\title{
OPTIMIZATION OF CONSUMPTION WITH PARTIAL OBSERVATION-JENSEN INEQUALITY METHOD
}

Abstract. A solution to a model of optimal consumption with partial observation considered in [LØS00] is presented. The approach is based on the Jensen inequality and does not require application of the filtering equation.

1. Introduction. We present a simplified solution of a generalized problem of optimal consumption in a dynamic setting with terminal conditions for the state process, related to the one considered in [LØS00] and originated in [M73] (in the case of complete observation and absence of state constraints). As in [LØS00], the problem is formulated in bayesian form where the unknown parameter is a random variable with known distribution. The model remains linear but replacement of the method based on the Kalman filter and stochastic maximum principle (see [YZ99]) by a simpler one - using the Jensen inequality and Pontryagin principle - allows us to extend the result of [LØS00] in three directions:

- the unobservable parameter is a process (not only a random variable),

- the observable parameters are not constant,

- a class of utility functions is considered.

Acknowledgements. The approach based on the Jensen inequality was suggested by Prof. Piotr Jaworski of Warsaw University to whom the author would like to express his gratitude. The article is a part of the author's Master Thesis [H03], written in Department of Mathematics, Warsaw University under the supervision of Prof. Łukasz Stettner, Institute of Mathematics, Polish Academy of Sciences.

2000 Mathematics Subject Classification: 93E11, 93E20, 60H10, 91B28, 91B70.

Key words and phrases: optimal consumption with partial observation, Jensen inequality, Pontryagin maximum principle. 
2. The main result. Let $\left(\mathbb{R}, \mathcal{F},\left\{\mathcal{F}_{t}\right\}_{t \in[0, T]}, \mathbf{P}\right)$ be a probability space with filtration, satisfying the usual conditions (see [YZ99]). By $\{W(t)\}_{t \in[0, T]}$ we denote an $\left\{\mathcal{F}_{t}\right\}_{t \in[0, T]}$-adapted Brownian motion process.

We consider an investor continuously consuming part of the value of a given portfolio and reinvesting the rest on the market. We assume that the theoretical value $\left\{X_{t}^{(c)}\right\}_{t \in[0, T]}$ of the investment portfolio with consumption which is observed by the investor is $\left\{\mathcal{F}_{t}\right\}_{t \in[0, T]}$-adapted and follows a stochastic differential equation of the form

$$
\begin{aligned}
d X^{(c)}(t)= & \left(A(t) X^{(c)}(t)+B(t) \mu(t)+g(t)-c(t)\right) d t \\
& +D(t) d W(t),
\end{aligned}
$$

where $X^{(c)}(0)=x_{0} \in \mathbb{R}, A, B, g, D \in C([0, T] ; \mathbb{R})$ and $\{\mu(t)\}_{t \in[0, T]}$ is an $\left\{\mathcal{F}_{t}\right\}_{t \in[0, T]}$-adapted process of unobserved parameter from $L^{1}(0, T ; \mathbb{R})$ and $\mu_{E}:[0, T] \rightarrow \mathbb{R}$ given by $\mu_{E}(t)=\mathbf{E} \mu(t)$ is continuous. The notation is consistent with [YZ99]. Partial observability means that the investor is only observing the filtration $\left\{\mathcal{G}_{t}\right\}_{t \in[0, T]}$ generated by the process $X$, which is not equivalent to observing the filtration $\left\{\mathcal{F}_{t}\right\}_{t \in[0, T]}$ for the trajectories of $\mu(t)$ are unknown. In this paper we do not consider the optimal investment problem. We restrict ourselves to finding an optimal consumption maximizing a given utility function.

The investor can freely choose the intensity of consumption, i.e. he chooses a process from the family

$$
\begin{gathered}
\mathcal{A}=\left\{c: \mathbb{R} \times[0, T] \rightarrow \mathbb{R}_{+} \mid \text {(i) } c \text { is }\left\{\mathcal{G}_{t}\right\}_{t \in[0, T]}\right. \text {-adapted, } \\
\text { (ii) } c \in L_{\mathcal{G}}^{1}(0, T ; \mathbb{R}) \cap L_{\mathcal{G}}^{2}(0, T ; \mathbb{R}), \\
\text { (iii) } c_{E}:[0, T] \rightarrow \mathbb{R}_{+} \text {and } c_{E}(t)=\mathbf{E} c(t) \text { is continuous, } \\
\text { (iv) } \left.\mathbf{E}\left[X^{c}(T)\right]=x_{T}\right\}
\end{gathered}
$$

for a given $x_{T}>0$. It is required that admissible consumption should guarantee the expected terminal value of portfolio. The problem is to maximize the measure of satisfaction of accumulated, discounted consumption. This means that for a given utility function $u: \mathbb{R}_{+} \rightarrow \mathbb{R}_{+}$with $u^{\prime}>0, u^{\prime \prime}<0$ we are interested in solving the following problem:

Problem 2.1. Find a process $c^{*} \in \mathcal{A}$ satisfying

$$
J\left(c^{*}\right)=\sup \{J(c): c \in \mathcal{A}\},
$$

where for given $\delta>0$ and $\gamma \in(0,1)$ the functional $J: \mathcal{A} \rightarrow \mathbb{R}$ is defined by

$$
J(c)=\mathbf{E}\left[\int_{0}^{T} e^{-\delta t} u(c(t)) d t\right] .
$$


THEOREM 2.1. Under the above assumptions the optimal consumption process $c^{*}$ for Problem 2.1 solves the following deterministic problem:

Find the intensity of consumption $c:[0, T] \rightarrow \mathbb{R}$, which is a continuous deterministic function of time such that $x^{(c)}(T)=x_{T}$, where $x^{(c)}$ solves

$$
\dot{x}^{(c)}(t)=A(t) x^{(c)}(t)+B(t) \mathbf{E}[\mu(t)]+g(t)-c(t), \quad x^{(c)}(0)=x_{0},
$$

and maximizes the functional $J$ defined by (4).

(i) Optimal intensity of consumption $c^{*}$ exists in the case of utility applied by [LØS00], i.e. assuming that $u(c)=c^{\gamma} / \gamma$ for given $\gamma \in(0,1)$, if

$$
x_{0} e^{-\chi(T)}-x_{T}+\int_{0}^{T} k(t) e^{-(\chi(T)-\chi(t))} d t \geq 0
$$

for

$$
\chi(t) \triangleq \int_{0}^{t} A(s) d s, \quad k(t) \triangleq B(t) \mathbf{E}[\mu(t)]+g(t)
$$

and is given explicitly by

$$
c^{*}(t)=\left(\lambda_{1} e^{-(\chi(T)-\chi(t))+\delta t}\right)^{1 /(\gamma-1)},
$$

where

$$
\lambda_{1}=\left(\frac{x_{0} e^{-\chi(T)}+\int_{0}^{T} k(t) e^{-(\chi(T)-\chi(t))} d t-x_{T}}{\int_{0}^{T} e^{-(\chi(T)-\chi(t))+\delta t} d t}\right)^{\gamma-1} .
$$

(ii) More generally, provided that inequality (6) holds, an optimal intensity of consumption $c^{*}$ exists if the utility function $u$ has the inverse multiplicativity property, i.e. there exists $I: \mathbb{R}_{+} \rightarrow \mathbb{R}_{+}$defined by $I(x) \triangleq$ $\left(u^{\prime}\right)^{-1}(x)$ such that

$$
\exists \kappa \in \mathbb{R} \forall x, y>0 \quad I(x y)=\kappa I(x) I(y) .
$$

Then

$$
c^{*}(t)=\kappa I\left(\lambda_{1}\right) I\left(e^{-(\chi(T)-\chi(t))+\delta t}\right)
$$

and

$$
\lambda_{1}=u^{\prime}\left(\frac{x_{0} e^{-\chi(T)}+\int_{0}^{T} k(t) e^{-(\chi(T)-\chi(t))} d t-x_{T}}{\kappa \int_{0}^{T} I\left(e^{-(\chi(T)-\chi(t))+\delta t}\right) e^{-(\chi(T)-\chi(t))} d t}\right) .
$$

Proof. We use the Jensen inequality and the Pontryagin maximum principle.

First we show that in the nonempty set of solutions of Problem 2.1 there is a deterministic function of time. By the Jensen inequality (see [KS97, The- 
orem $2, \S 5.7])$, for $c \in \mathcal{A}$ we have

$$
\mathbf{E} \int_{0}^{T} e^{-\delta t} \frac{c(t)^{\gamma}}{\gamma} d t=\int_{0}^{T} \mathbf{E}\left[e^{-\delta t} \frac{c(t)^{\gamma}}{\gamma}\right] d t \leq \int_{0}^{T} e^{-\delta t} \frac{[\mathbf{E} c(t)]^{\gamma}}{\gamma} d t .
$$

Then if $c(t) \in \mathcal{A}$ is a solution, we can write $c=\mathbf{E} c(t)+c_{0}(t)$, where $c_{0}(t)$ is random, different from 0 on a set of positive measure and $\mathbf{E} c_{0}(t)=0$. So if $J$ is maximized and we take as control the function $\mathbf{E} c(t)$ instead of the process $c(t)$ the value of the functional does not decrease. That is what was claimed.

On the other hand, there exists a unique solution of (1) for every $c \in \mathcal{A}$ (conclusion from [YZ99, Chapter 2, Theorem 5.2]) because of the regularity conditions (see [YZ99, Chapter 1, §6.4]) of the drift term $b:[0, T] \times \mathbb{R} \times$ $\mathbb{R}_{+} \times \mathbb{R} \rightarrow \mathbb{R}$

$$
b(t, x, c, \omega) \triangleq A(t) x+\mu(t, \omega) B(t)+g(t)-c,
$$

and $c \in L_{\mathcal{G}}^{2}(0, T ; \mathbb{R})$. But $\mu_{E} \in C([0, T] ; \mathbb{R})$. Since $D$ is deterministic and square integrable the process $\int D(s) d W(s)$ is a martingale (e.g. being an Itô integral). Integrating (1), taking expectation $\mathbf{E}$ and applying the Fubini Theorem (see [KS97]) to the integral

$$
\mathbf{E}\left[\int_{0}^{t} B(s) \mu(s) d s\right]
$$

we conclude that $c^{*}$ has to satisfy (5).

To finish the proof we have to show (i) and (ii). Because part (i) is a special case of (ii) we only consider the case of (ii) and prove (10). It is easy to show that the utility function $\left(u(c)=c^{\gamma} / \gamma\right)$ satisfies (9). We use the Pontryagin Principle (see [YZ99, Chapter 3, Theorem 2.1]). The Hamiltonian for this optimization problem is

$$
H(t, x, c, p)=p(A(t) x+B(t) \mathbf{E}[\mu(t)]+g(t)-c)+e^{-\delta t} u(c(t))
$$

and $H$ is maximized with respect to $c$ if

$$
-p(t)+e^{-\delta t} u^{\prime}(c(t))=0
$$

On the other hand, the adjoint equation of the Maximum Principle leads to

$$
\left\{\begin{array}{l}
\dot{p}(t)=A(t) p(t) \\
p(T)=\lambda_{1}
\end{array}\right.
$$

that is,

$$
\forall t \in[0, T], \quad p(t)=\lambda_{1} e^{-\int_{t}^{T} A(s) d s} .
$$

Combining (13) and (14), and applying the assumed property (9) of the 
function $I$ (well-defined since $u^{\prime \prime}<0$ ) we obtain

$$
c^{*}(t)=I\left(\lambda_{1} e^{\int_{t}^{T} A(s) d s+\delta t}\right)=\kappa I\left(\lambda_{1}\right) I\left(e^{\int_{t}^{T} A(s) d s+\delta t}\right) .
$$

To find $\lambda_{1}$ we use (5) integrated over $[0, T]$ and the terminal condition $\mathbf{E} X^{c^{*}}(T)=x_{T}$ as follows:

$$
\begin{aligned}
& x_{T}= x_{0} e^{-\int_{t}^{T} A(s) d s}+\int_{0}^{T}\left(B(t) \mathbf{E}[\mu(t)]+g(t)-c^{*}(t)\right) e^{-\int_{t}^{T} A(\tau) d \tau} d t, \\
& x_{T}=x_{0} e^{-\chi(T)}+\int_{0}^{T} k(t) e^{-(\chi(T)-\chi(\tau))} d t \\
& \quad-\kappa I\left(\lambda_{1}\right) \int_{0}^{T} I\left(e^{\chi(T)-\chi(t))+\delta t}\right) e^{-\chi(T)-\chi(t))} d t,
\end{aligned}
$$

which after transformations leads to (11). The assumption (6) ensures that $\lambda_{1}$ is nonnegative, which is required by the transversality condition (see [YZ99, Chapter 3, Theorem 6.1, Equation (6.6)]).

REMARK 2.1. The inverse multiplicativity property is not very restrictive (see [C91]). The utilities $u(c)=\ln c$ and $u(c)=c^{\gamma} / \gamma$ have this property.

REMARK 2.2. The result of Theorem 2.1 extends the class of problems from [LØS00] that do not have the certainty equivalence property. It should be emphasized that the way of solving the problem avoids partial observability difficulties. Existence of the unique solution to (5) requires stronger assumptions on the parameters of (1) than those made in [LØS00]. However, in both cases, the optimal solution is deterministic and continuous.

On the other hand, broad applicability of such an approach is doubtful; examples considered in [H03], however simple and more realistic, cannot be reduced to the deterministic case in that way.

\section{References}

[C91] J. W. Coleman, Equilibrium in production economy with income tax, Econometrica 59 (1991), 1091-1104.

[H03] G. Hałaj, Partially observed problems in mathematics of finance, Master Thesis, Dept. Math., Warsaw Univ., 2003 (in Polish).

[KS97] I. Karatzas and S. E. Shreve, Brownian Motion and Stochastic Calculus, Springer, New York, 1997.

[LØS00] D. Lefèvre, B. Øksendal and A. Sulem, An introduction to optimal consumption with partial observation, working paper, November 2000.

[M73] R. Merton, Optimal consumption and portfolio rules in a continuous time model, Economic Theory 3 (1971), 373-413. 
[YZ99] J. Yong and X. Zhou, Stochastic Controls. Hamiltonian Systems and HJB Equations, Springer, New York, 1999.

Naukowa $9 / 14$

02-469 Warszawa, Poland

E-mail: grzegorz.halaj@n-s.pl

Received on 31.1.2004;

revised version on 7.7 .2004

(1740) 